livraisons

d'Histoire

de l'Architecture

\section{Livraisons de l'histoire de l'architecture}

34 | 2017

Le bois

\title{
Une vision économique du bois chez les théoriciens des Temps modernes? Une vision pratique
}

"An economic vision of wood among modern theorists? A practical vision" "Eine wirtschaftliche Perspektive des Holzes bei den Theoretikern der modernen Epoche? Eine praxisorientierte Sichtweise“

\section{Hélène Rousseau-Chambon}

\section{(2) OpenEdition}

\section{Journals}

Édition électronique

URL : http://journals.openedition.org/lha/782

DOI : 10.4000//ha.782

ISSN : 1960-5994

Éditeur

Association Livraisons d'histoire de l'architecture - LHA

Édition imprimée

Date de publication : 15 décembre 2017

Pagination : $9-15$

ISSN : 1627-4970

\section{Référence électronique}

Hélène Rousseau-Chambon, " Une vision économique du bois chez les théoriciens des Temps

modernes? Une vision pratique », Livraisons de l'histoire de l'architecture [En ligne], 34 | 2017, mis en ligne le 15 décembre 2019, consulté le 25 mars 2020. URL : http://journals.openedition.org//ha/782 ; DOI : https://doi.org/10.4000/lha.782 


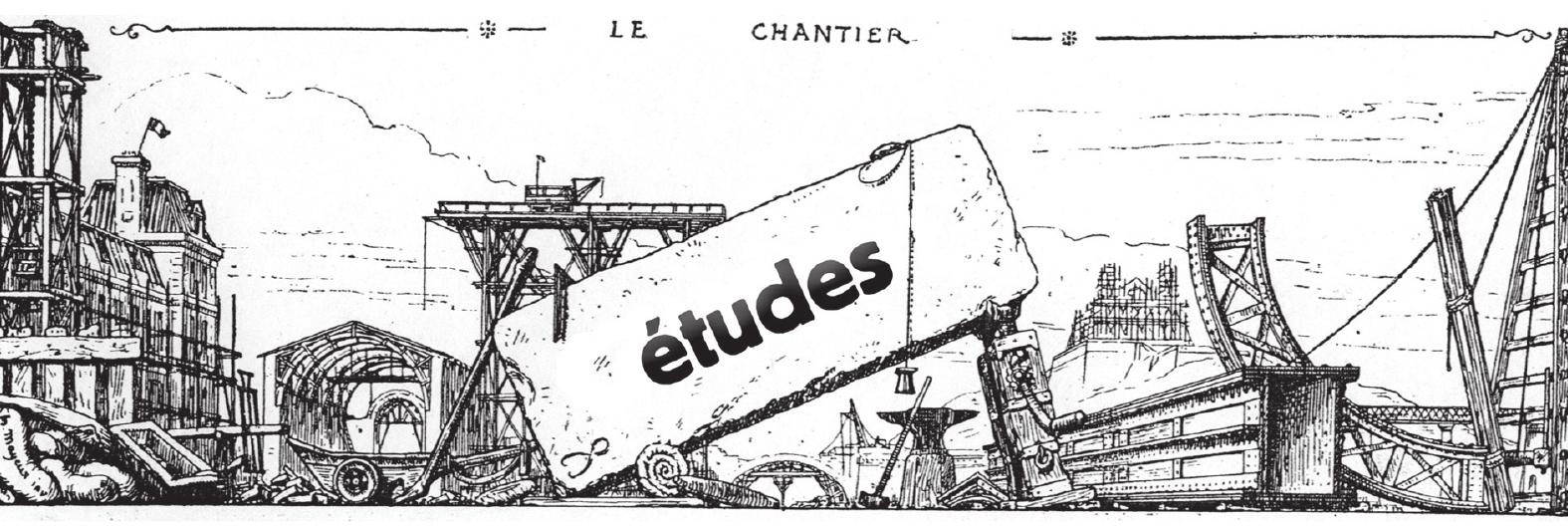





\section{UNE VISION ÉCONOMIQUE DU BOIS CHEZ LES THÉORICIENS DES TEMPS MODERNES ? UNE VISION PRATIQUE}

Dans un article paru en 1991, J.-M. Pérouse de Montclos s'était penché sur le lien, au cours des Temps modernes, entre approvisionnement en bois et innovations techniques dans la charpente, un des éléments du bâti le plus gourmand en bois ${ }^{1}$. Il montrait combien l'absence de bois de grande portée avait conduit à une évolution technique au XVIII ${ }^{\mathrm{e}}$ siècle, phénomène admis généralement aujourd'hui. Depuis, Rachel Touzé a relevé les thématiques récurrentes dans les textes théoriques des Temps modernes - qualité des bois, essences, période de coupe notamment ${ }^{2}$. En effet, tout architecte devait avoir des connaissances quant aux essences les plus à même d'être utilisées dans l'architecture, leur coupe qui dépend de la saison, de l'âge du bois et de la lunaison, et leurs usages dans l'architecture - la terminologie spécifique à la charpenterie étant alors précisée ; les théoriciens se démarquaient d'ailleurs parfois des propositions de Vitruve et des théoriciens italiens de la Renaissance. Mais Rachel Touzé ne s'est intéressée ni à la mise en œuvre de ce matériau, thématique fondamentale pour l'histoire de la construction, ni aux aspects économiques du bois.

Or, si les théoriciens de l'architecture évoquent certes plus rarement les aspects économiques du bois que la nature de ce matériau, il n'en demeure pas moins que de Philibert de l'Orme à Henri Louis Duhamel du Monceau, nombreux sont les théoriciens qui entendent se pencher sur le coût réel de la construction en bois. En outre à ces théoriciens, il faut ajouter les membres de l'Académie royale d'architecture qui, à partir de 1671, ne manquent pas de donner leur avis sur tout ce qui touche à l'architecture, et notamment sur la mise en œuvre du bois, lors des conférences académiques ${ }^{3}$. Il faut aussi tenir compte des conseils délivrés par les professeurs de l'Académie d'architecture à leurs élèves quant aux aspects pratiques de la mise en œuvre de ce matériau. Ce sont autant de sources qui sont rarement mises

1. Jean-Marie Pérouse de Montclos, "Difficultés d'approvisionnement en grands bois et innovations techniques dans la charpente française des Temps modernes ", J.-L. Biguet, J. Boissière, J.-C. Huvé, Le bois et la ville du Moyen Âge au XXe siècle, ENS Fontenay, 1991, p. 223-235.

2. Rachel Touzé, "Les traités d'architecture. Le bois dans les traités d'architecture du $X^{\mathrm{e}}$ au XVIII ${ }^{e}$ siècle : entre l'antique et le moderne ", Les Charpentes $d u X I^{e}$ au XIX ${ }^{e}$ siècle, Grand Ouest de la France, Typologie et évolution, analyse de la documentation de la médiathèque de l'architecture et du patrimoine, Brepols, 2011, p. 21-27.

3. Hélène Rousteau-Chambon, "Le bois dans les discours académiques (1671-1792) ", $2^{e}$ congrès francophone d'histoire de la construction, Lyon, 29-31 janvier 2014, Les Temps de la construction, Processus, acteurs, matériaux, Paris, Picard, 2016, p. 153-162. 
en relation mais qui permettent d'avoir une vision générale de l'économie du bois, d'un point de vue théorique tout du moins, qui va de pair avec une normalisation des savoirs, démarche constante de la théorie architecturale des Temps modernes.

Cet intérêt pour le bois est aussi intimement lié à la recherche d'une meilleure gestion des forêts, le bois étant indispensable tant pour la marine en plein développement que pour les demeures plus nombreuses du fait de la croissance démographique; le bois de chauffage faisant quant à lui l'objet d'une législation spécifique. Aussi pour répondre à cette préoccupation, des outils géométriques sont utilisés, des devis de construction précis se développent pour protéger aussi bien le maître d'ouvrage que le maître d'œuvre, et des utilisations différentes du bois sont prônées, en se fondant cette fois plus spécifiquement sur la résistance des matériaux.

\section{Mesurer le bois pour une plus grande exactitude}

Les architectes des Temps modernes ont une vision toujours très pratique des règles architecturales. Le plus souvent c'est par l'exemple que se pratiquent une généralisation et la recherche d'une norme. Il n'est donc pas étonnant que de manière très pragmatique, Savot, en 1624, après avoir livré les grands principes d'une heureuse distribution, se penche longuement sur le coût des matériaux de construction. Pour la charpenterie notamment, il commence par expliquer la manière d'acheter le bois : "Le bois de charpenterie se vend au cent de pièces. La pièce doit avoir douze pieds de long, \& six pouces en quarré, tellement qu'elle contient trente six pouces sur douze pieds de longueur ${ }^{4}$. » Le propos de Savot reste très général puisqu'il n'envisage qu'une longueur de bois - un demi-siècle plus tard, Félibien reprend ces propos ${ }^{5}$. Or, déjà les poutres de bois peuvent être de longueur variée. Il est admis en effet, selon les us et coutume de Paris, comme l'explique Mathurin Jousse au milieu du XVII ${ }^{\mathrm{e}}$ siècle, qu'elles soient :
«De 6 pi. 9 pi. 12 pi. 18 pi, 21, 24, 27, 30, 36, 39, 42. C’est pourquoy lorsque dans un toisé qui est fait suivant les us et coutumes, on a des bois plus petits que quelques unes des grandeurs cy- dessus, on les conte [sic] toujours de la grandeur la plus proche superieure, à moins qu'ils ne se trouvent faire quelque partie juste de celles que nous avons marqué ; car une piece de bois d'un pied sera conté $[$ sic] pour un pied \& demy, parce qu'il est le quart de $6 \mathrm{pi}^{6}$."

Et le charpentier théoricien livre un tableau qui explicite ses dires. Dans la seconde moitié du XVIII siècle, J.-F. Blondel reprend ces principes, sans se référer expressé-

4. Louis Savot, L'Architecture françoise des bastimens particuliers, Paris, S. Cramoisy, 1624, p. 288.

5. André Félibien, Des principes de l'architecture, de la sculpture, de la peinture et des autres arts qui en dépendent, Paris, J.-B. Coignard, 1676, p. 117.

6. L'Art de la charpenterie (1702) de Mathurin Jousse, corrigé et augmenté de ce qu'il y a de plus curieux dans cet art et des machines les plus nécessaires à un charpentier par M. D.L. H, le tout enrichi de figures et de tailles douces, Paris, de la Moette, 1702 [1 $1^{\text {re }}$ éd. de M. Jousse, 1627], p. 193. 
ment à la coutume cependant ${ }^{7}$. Tout au long des Temps modernes, il appartient donc au charpentier d'utiliser au mieux les coupes de bois pour avoir le moins de chutes. On comprend alors davantage pourquoi les théoriciens, du XVII ${ }^{\mathrm{e}}$ siècle surtout, lient ces propos à une description de chaque pièce de charpente, et à leur usage. Si savoir toiser le bois lors de son achat est fondamental, il est tout aussi important de pouvoir pratiquer ces mesures lors de la mise en œuvre du matériau. Selon Jousse, pour éviter tout problème de mesure, le particulier doit " toiser les grosseurs et longueurs mises en œuvre ${ }^{8}$ ". Déjà Savot, s'appuyant sur une démarche très pratique, avait expliqué clairement la manière de toiser :

"L'on a jugé à propos pour la facilité de ce toiser de diviser ce qu'on appelle une pièce de bois que l'on peut concevoir comme un morceau de poutre qui auroit un pied de gros sur trois pieds de long en 144 chevilles, d'un pouce de gros sur trois pieds de long, \& qui contiendront par conséquent 36 pouces cubes. Il faudra 9 de ces chevilles pour faire $1 / 16$ de pièce, 18 pour faire $1 / 8$ de piece, 36 pour faire $1 / 4,72$ pour faire $1 / 2,108$ pour faire $3 / 4$ $\& 144$ comme nous l'avons dit pour une pièce ${ }^{9}$. "

La démarche semble simple puisqu'elle est fondée sur une suite de fractions, mais toutes les pièces de bois n'ont pas le même diamètre, ni la même dimension. Aussi pour rendre ses propos plus clairs, il fournit un tableau assez complexe. Mais le maniement de ce catalogue reste mal aisé et peu adapté aux réalités de la construction. Par conséquent, pour établir des règles qui seront ensuite mises en pratique, il faut faire appel aux apports de la géométrie. Or dans les premières années du XVII ${ }^{\mathrm{e}}$ siècle, des mathématiciens publient des ouvrages mêlant arithmétique et géométrie pratique. Ainsi, on doit à Jean Abraham, en 1617, la publication d'un ouvrage dans lequel sont associés construction et quantitatif, ce qui permet d'estimer les volumes d'approvisionnement nécessaires à la construction ${ }^{10}$. De même, les livres de F. Le Gendre et de Barrême ${ }^{11}$ permettent de mettre en application plus aisément

7. Jacques-François Blondel, Cours d'architecture civile ou Traité de la décoration, distribution et construction des bâtiments contenant les leçons données en 1750 et les années suivantes, Paris, Desaint, 1771-1777, 6 vol. Le tome VI est de Pierre Patte. L'auteur se fonde une nouvelle fois sur la pratique. En effet explique-t-il, les coupes des arbres peuvent se faire selon différentes dimensions (p. 232) : "l'on coupe les bois dans les forêts suivant une progression de 3 pieds en 3 pieds, c'est à dire selon une longueur constante de 3, 6, 9, 12, 15 pieds, etc. \& comme le Charpentier les achète en conséquence de ces longueurs prescrites, il convient donc [...] d'y avoir égard, \& de prendre le nombre en sus pour lui en tenir compte [...]".

8. L'Art de la charpenterie (1702) de Mathurin Jousse, op. cit.

9. Louis Savot, op. cit., p. 193.

10. L'Arithmétique, arpentage universel, toise des bastimens, géométrie inaccessible, géométrie par la règle et le compas, avec la fabrique et usage des quadrans sollaires, composé par Jean Abraham, dit Launay, Rouen, P. L'Oyselet, 1617.

11. François Le Gendre, L'Arithmétique en sa perfection, mise en pratique selon l'usage des financiers, banquiers et marchands, avec un traité de géométrie pratique appliquée à l'arpentage et un abrégé d'algèbre, Paris, s.l., 1657 ; 1668 ; L'arithmétique du sr Barreme ou le livre facile pour apprendre l'arithmétique de soi-même, 1673. 
les théorèmes de Pythagore et de Thalès, les égalités angulaires, ou la géométrie dans l'espace. Grâce à l'apport de ces divulgateurs scientifiques, P. Bullet peut, dans son Architecture pratique (1691), intégrer «les données élémentaires de géométrie et de trigonométrie dans le quotidien des agences ${ }^{12}$ ». Bullet en effet, commence par donner des méthodes de calcul pour mesurer des superficies planes et des corps solides avant de livrer un processus pour toiser des éléments architecturaux variés (des voûtes aux formes diverses aux cheminées et ornements) et d'expliquer la manière de faire des calculs pour estimer justement les pièces de bois ${ }^{13}$. Cette fois, il ne livre d'ailleurs pas de tableau, ne souhaitant sans doute pas simplifier indûment les calculs, mais se contente d'expliquer le toiser géométriquement. Bullet entend mathématiser le savoir de l'architecte. En 1755, cependant, dans une nouvelle édition largement augmentée de ce texte qui connaît un grand succès, Descouture, un ancien inspecteur toiseur, ajoute un tableau gravé en taille douce de la grosseur des bois sur une toise de longueur ${ }^{14}$. Il apparait donc impératif, compte tenu des calculs somme toute assez complexes, de fournir un tableau à la lecture plus immédiate. C'est d'ailleurs à cette table que se réfere Blondel dans le chapitre consacré à la réduction des bois de charpente ${ }^{15}$. Les architectes, toiseurs ou experts géomètres n'ont de fait alors qu'une aisance très relative dans les chiffres.

Pour estimer précisément les coûts de construction, il faut donc pouvoir mesurer, toiser exactement les différentes pièces de bois, opération qui n'est pas aisée à mettre en place alors, les connaissances en géométrie restant très basiques. Il n'empêche qu'achat de bois et mise en œuvre sont intimement liés.

\section{Des cô̂ts de construction estimés plus justement}

Depuis Philibert de L'Orme, bâtir à l'économie reste un maître mot de la construction, et l'architecte comme l'entrepreneur sont ceux qui connaissent le mieux le coût réel des matériaux et de leur mise en ouvre ${ }^{16}$. Le devis constitue un outil juridique qui permet de coucher par écrit ce coût de construction, notamment celui du bois. Si Jacques Androuet du Cerceau ${ }^{17}$ et Louis Savot ${ }^{18}$ donnent quelques indications quant au métré de quelques pièces, c'est surtout Pierre Bullet qui, pour la première fois, livre des indications précises, un véritable cahier des charges, pour l'écriture d'un devis. Il donne un modèle de devis général pour "les ouvrages de

12. Pierre Bullet, L'architecture pratique, [Paris, 1691], introduction de Thierry Verdier, Montpellier, Presses universitaires, 2006, p. VII.

13. Pierre Bullet, ibid., "Du toisé des bois de charpenterie ", p. 240-248.

14. Annie Charron, "L'architecture pratique de Pierre Bullet ", La Construction savante, les avatars de la littérature technique, Paris, Picard, 2008, p. 55-71.

15. Jacques-François Blondel, op. cit., t. VI, p. 232.

16. Robert Carvais, "Une relecture de l'œuvre écrite de Philibert de L’Orme ou la résolution de l'expertise ", Les Temps de la construction, processus, acteurs, matériaux, Paris, Picard, 2016, t. I, p. 226-237.

17. Jacques Androuet du Cerceau, Les Plus excellents bâtiments de France, Paris, Gilles Beys, 1576.

18. Louis Savot, op. cit.. 
maçonnerie, charpenterie, couverture menuiserie, ferrure, et gros fer, vitrerie, pavé de grais $\&$ peinture d'impression ${ }^{19}$ " avant d'établir des modèles spécifiques pour la charpenterie et la couverture. Bullet révèle ainsi les particularités de ce matériau dont les modes de calcul ne sont pas communs à ceux de la pierre, et la difficulté, pour l'architecte, de maîtriser des coûts dans un domaine qu'il connaît moins bien - les charpentiers constituent un corps indépendant de celui des maçons et architectes. Aussi trouve-t-il nécessaire d'expliquer: "Quand on fait un devis pour la charpenterie, on doit y marquer d'abord l'espèce $\&$ la qualité des bois que l'on doit employer ", rappelant par là même que le coût d'une charpente dépend des matériaux utilisés, comme pour tout autre partie de l'édifice. Il poursuit : il faut «[...] commencer par la charpente des combles \& tout ce qui doit y avoir rapport, ensuite les planchers, les cloisons, les escaliers \&ct [...]». Par cette liste, Bullet entend montrer la démarche systématique que doit mener tout architecte soucieux d'établir un devis précis. Puis il continue : "Il faut aussi marquer dans chaque espece d'ouvrage la grosseur des bois qu'on y doit employer \& ceux qui doivent estre de brin ou de sciage ", tout en précisant l'endroit précis où ils seront utilisés. La taille $\mathrm{du}$ bois et sa coupe sont fondamentales puisque seul le bois de brin peut convenir aux charpentes et aux solives des plafonds ${ }^{20}$. Bullet ajoute à cette définition générale, des modèles de devis pour les combles, les planchers, les cloisons et pans de bois. À une époque où les prix faits sont essentiellement rédigés "à la manière d'une visite quasi virtuelle ${ }^{21}$ ", on comprend mieux l'apport des devis donnés en exemple par Bullet. Ces devis correspondent à la codification d'une pratique et permettent d'établir des règles juridiques appliquées à la construction ${ }^{22}$. Quelques années plus tard, ils sont assez largement adoptés puisque D’Aviler en livre une définition précise : "C'est un mémoire général des quantités, qualités \& façons des matériaux d'un bâtiment, fait sur des dessins cotés $\&$ expliqués en détail, avec les prix de chaque espece d'ouvrage, par toise ou par tâche, sur lequel l'Entrepreneur marchande \& convient avec le Bourgeois d'exécuter l'ouvrage, moyennant une certaine somme [... ${ }^{23}$ ». Les devis comme le toisé constituent des outils juridiques et mathématiques qui montrent une volonté non seulement de codifier l'architecture, mais d'en maîtriser les coûts plus parfaitement. Économie et théorie de la construction sont dont intimement liées au cours des Temps modernes.

19. Pierre Bullet, op. cit., p. 354.

20. Augustin-Charles D'Aviler, Cours d'architecture (...) avec une ample explication des termes d'architecture, 1691, $2^{\mathrm{e}}$ éd. revue et augmentée par Le Blond, 1710, p. 65 : "bois de brin \& de tige. Bois dont on a seulement ôté les quatre dosses flaches pour l'équarrir \& qui sert pour les combles, les poteaux corniers, les pans de bois, $\&$ les solives des planchers". Bois de sciage "bois qui est propre à refendre ou qui est débité à la scie en chevrons, membrures ou planches ».

21. Pierre Bullet, op. cit., [Paris, 1691], introduction de Thierry Verdier, p. IV.

22. Sur ce sujet, voir Juliette Hernu-Belaud, "The architecture pratique by Pierre Bullet 1691] and the Normalisation of the Construction Process ", Nuts and bolts of construction history, culture, technology and society, R. Carvais, A. Guillerme, V. Nègre, J. Sakarovitch éd, t. 1, p. 489-502.

23. Augustin-Charles D’Aviler, op. cit., p. 148. 


\section{Une mise en ouvre à l'économie}

Établir des règles ne suffit pas pour avoir une construction plus régulière. Il faut aussi savoir utiliser les pièces de bois qui conviennent non seulement le mieux à la solidité du bâti mais aussi à maîtriser le coût de construction. L'étude de la résistance des matériaux devient alors fondamentale. Déjà Mariotte dans le Traité du mouvement des eaux (1686), publié par Philippe de la Hire, en avait rappelé quelques principes. Il n'est donc pas étonnant que ce dernier, devenu professeur de l'Académie royale d'architecture, ait essayé d'apporter des données précises à ses élèves dans ses cours d'architecture dispensés à deux reprises (en 1705-1706 puis en 1714-1715). La Hire essaie en effet d'établir une règle pour trouver la grosseur des poutres suivant leur portée et leur longueur qu'il illustre par un exemple, selon son habitude : "il faut trouver la superficie de l'extremité d'une poutre qui a 36 pieds de longueur. Au lieu de 36 pieds, je prens 36 pouces suivant la règle et j'en tire la racine carrée qui est 6.4 fois 6 est 24 pour l'un des costé du bout de la poutre. Mais la $1 / 2$ de 36 est 18 . On aura donc 18 pouces pour l'autre coté et le produit qui vient la multiplication de 18 par 24 est 432 pouces de superficie pour celle du but de la poutre ${ }^{24}$ ». La description est simple mais longue du fait de l'absence de signes algébriques qui se développent surtout dans la seconde moitié du siècle. À la suite de cet exposé, et pour rendre les applications de ses propos plus aisés, La Hire livre une "table des superficies des poutres par leurs extrémités sur les longueurs depuis 8 pieds jusqu’à 36 ». Bien que Philippe de la Hire soit un brillant mathématicien - il est aussi membre de l'Académie des sciences et enseigne la géométrie au Collège Royal -, pour lui, les règles de mathématiques doivent pouvoir s'appliquer aisément à l'architecture. Outre ce tableau pourtant très clair, il livre un exemple concret propre à la mise en œuvre du bois : "il n'est pas necessaire de faire toujours ces pieces de bois de la grosseur qu'on leur trouve par cette règle à moins qu'elles ne servent à porter un fardeau très considérable comme celui d'un plancher ${ }^{25}$ ". Le pragmatisme et l'économie des matériaux priment. Cette recherche se retrouve d'ailleurs explicitement dans les procès-verbaux des séances académiques puisque le 30 janvier 1688, les académiciens s'entretiennent sur la poussée et la pesanteur des combles et, disent-ils, " on a résolu de dresser un tarif de la grosseur des bois propres à employer tant pour les combles que pour les planchers et cloisons ${ }^{26}$ ", car les ouvriers "font souvent, tant par ignorance que par intérest " de mauvais calculs. C'est Philippe de la Hire qui présente ce "tarif» les 20 et 22 février de la même année.

Le prix du bois varie en fonction de la taille de la poutre mais les pièces de très grande taille, outre leur grand prix ne sont pas sans poser de problèmes : d'une part, elles occasionnent un poids énorme sur l'édifice, et le fragilisent donc, et d'autre part, elles correspondent à des bois plus vieux, plus souvent "viciés et imparfaits ",

24. B. Institut, ms 8125 , fo $216 \mathrm{v}$.

25. Ibid.

26. Procès-verbaux de l'Académie royale d'architecture, publiés sous la dir. de Henry Lemonnier, Paris, Jean Schelit, Édouard Champion puis Armand Colin, 1911-1926, 10 vol., t. III, p. 155. 
ce qui rend la stabilité encore plus hypothétique. Aussi, depuis le début du XVIII ${ }^{e}$ siècle surtout, les théoriciens prônent les assemblages de différentes poutres. Déjà, Philippe de la Hire explique dans son cours : "On ne trouve pas toujours les pièces de bois aussi longues qu'il est nécessaire dans quelque bastiments. C'est pourquoi on a cherché un moyen de composer plusieurs et de leur donner autant qu'il est possible une très grande solidité». Cette pratique avait déjà été expliquée longuement par Philibert de L'Orme ${ }^{27}$, sans véritablement être mise en œuvre. Il faut attendre J.-F. Blondel pour qu'elle soit généralisée dans la théorie, ce dernier expliquant lapidairement que «l'expérience confirme qu'il vaut mieux placer deux moyennes poutres à côté l'une de l'autre qu'une seule de forte qualité car alors chaque poutre ne porte que la moitié du poids de la travée d'un plancher, au lieu qu'une seule en porte deux ${ }^{28}$ ". Une construction en assemblage peut donc être plus solide et moins chère qu'une construction d'un seul tenant.

La construction en bois reste empirique au cours des Temps modernes. Cependant, des théoriciens cherchent alors à comprendre la mise en œuvre de ce matériau pour la mettre en pratique plus aisément, voire pour l'organiser. Cette réflexion nouvelle couchée sur le papier passe par une meilleure connaissance constructive des matériaux et de leur mise en œuvre. Elle va de pair aussi avec le développement de nouveaux outils mathématiques et juridiques (toisé, devis) qui sont mis à la disposition des hommes de l'art, et parallèlement, à l'organisation de métiers, les experts et les toiseurs qui sont chargés de vérifier la construction. Tout concourt donc à mieux connaître les coûts de construction, préalable indispensable à une préindustrialisation du matériau, dans un second temps, au siècle suivant.

Hélène ROUSTEAu-CHAMBON professeur d'histoire de l'art moderne, université de Nantes

27. Philibert de L'Orme, Nouvelles inventions pour bastir à petitz fraiz trouvées naguères par Philibert de L'Orme [...], Paris, impr. F. Morel, 1561.

28. Jacques-François Blondel, op. cit., p. 240. 
